\title{
Un autoanálisis crítico para docentes de la enseñanza bilingüe: Ideología, pedagogía, acceso y equidad (IPAE)
}

\author{
A Critical Self Examination for Dual Language Educators: \\ Ideology, Pedagogy, Access, and Equity (IPAE)
}

Cristina Alfaro y Ana Hernández ${ }^{1}$

\section{Resumen}

El crecimiento exponencial de los programas de Educación de Lenguaje Dual (DLE) en los Estados Unidos ha hecho aún más evidente la apremiante necesidad de auto examiner críticamente la conciencia didáctica y fundamental sobre la equidad y la justicia social (Darder, 2015; Valenzuela, 2016). Un autoanálisis con el marco teórico que incluye los cuatro principios en la Ideología, Pedagogía, Acceso y Equidad (IPAE) puede impulsar a los educadores a examinar preguntas arduas que causan reflexión y razonamiento sobre sus creencias y didácticas. Históricamente, dadas ideologías han existido enraizadas profundamente en el monolingüismo, la asimilación y la falta de respeto al estudiante bilingüe. En cambio atribuye a la preparación del docente y al desarrollo profesional sobre la claridad ideological, el propósito pedagógico, el acceso a la educación y la transformación de la equidad en el plan de estudios o procesos de instrucción. En este artículo afirmamos como la conciencia crítica y la praxis conducen la equidad y son equivalentemente importantes en la formación docente, inclusivamente su conocimiento de técnicas y metodología para la alfabetización bilingüe.

\section{Palabras clave}

Ideología, pedagogía, acceso, equidad, conciencia crítica.

\section{Abstract}

The extremely rapid growth of Dual Language Education (DLE) programs across the nation has made even more evident the pressing need for self-examination of practices that are critically conscious and fundamental to equity and social justice (Darder, 2015; Valenzuela, 2016). Examining the four tenets Ideology, Pedagogy, Access and Equity (IPAE) can propel educators to potentially ask the tough questions that cause reflection and recalibration. Given historical and deeply engrained English monolingual, assimilationist, and deficit ideologies regarding linguistically diverse students, it is incumbent for DLE teacher preparation and professional development to address the role of ideological clarity, pedagogical clarity, access for all, and equity in shaping the curriculum and instructional processes. In this article we argue that equally as important to a teachers' knowledge of technical and biliteracy methodological skills is their critical consciousness and praxis that leads to educational equity.

\section{Keyworks}

Ideology, pedagogy, access, equity, critical consciousness. 


\section{Introducción}

Debido al crecimiento de los estudiantes de prekinder a $12 .^{\circ}$ grado, el crecimiento exponencial de los programas bilingües, y la gentrificación de la educación en dos idiomas, la necesidad de docentes bilingües con conciencia crítica es innegablemente vital ahora más que nunca (Alfaro, 2017; Alfaro \& Bartolomé, 2018; Freire, 2016). Los programas de educación bilingüe continúan creciendo de una manera exponencial en los Estados Unidos, particularmente en el estado de California donde una tercera parte de los programas existen (Center for Applied Linguistics, 2017). A causa de este crecimiento, se ha dado la necesidad de crear cursos de capacitación para crear el conocimiento de los docentes sobre la educación bilingüe (Alfaro \& Bartolomé, 2018; Bartolomé, 2010; Gándara \& Maxwell-Jolly, 2000; Darder, 2015; Garcia; 2014; Lewis, Jones, \& Baker, 2012, 2013). Nosotras vemos este reto como un tema fundamental en lo que conlleva a la equidad y justicia social, dada nuestra perspectiva que los docentes puedan anunciar o denunciar las prácticas equitativas dentro del aula (Alfaro et al., 2015; Bartolomé, 2008), que por causa podría afectar la eficacia de las experiencias educativas de los estudiantes bilingües (Cazden, 2001). Nosotras escribimos desde la perspectiva privilegiada de formadoras de docentes e investigadoras, así como docentes bilingües y administradoras con más de treinta años de experiencia en el área de la educación bilingüe. En este artículo nuestra intención es dialogar sobre la necesidad de apoyar a los docentes en la educación bilingüe e inglés como segundo idioma, o mejor conocido por sus iniciales en inglés como DLE (Dual Language Education), para que ellos puedan tener un mejor entendimiento de los cuatro temas del autoanálisis: Ideología, Pedagogía, Acceso, Equidad (IPAE), que son necesarios para fortalecer de una manera critica la enseñanza en las aulas de DLE.

Los cursos de capacitación para los docentes bilingües típicamente se enfocan en la metodología para desarrollar un alto nivel de bilingüismo, que incluye el alfabetismo y la cognición en ambas lenguas para todos los estudiantes (Alfaro, et al., 2015; Baker, 2011, 2006; Quezada \& Alfaro, 2012; Wright et al., 2015). Mientras tanto las investigaciones indican que un programa bilingüe de adecuada implementación promueve el éxito académico (Callahan \& Gandara, 2014; Genesee, et al., 2009; Soltero, 2016), ya que sabemos muy poco sobre los docentes bilingües y de la manera en que implementan intencionalmente y estratégicamente los siguientes temas en sus programas: Ideología, Pedagogía, Acceso, Equidad (IPAE). En un informe titulado: Preguntas urgentes sobre la investigación y la problemática en la enseñanza de dos idiomas ('Urgent Research Questions and Issues in Dual-language Education') (Parkes et al., 2009) se presentaron algunas de estas comunes preocupaciones y preguntas en la exanimación de temas. Dadas estas circunstancias sobre la escolaridad de los docentes, estamos en el deber de pensar profundamente sobre la importancia del marco teórico de IPAE en la implementación efectiva de los programas bilingües (DLE Programs).

La realidad es que los docentes bilingües enseñarán en aulas donde al menos, la mitad de sus estudiantes vendrán de un estatus social de punto bajo (Socio-Economic Status - SES), no serán de la raza blanca o mayoritaria, sino vendrán de grupos culturales que históricamente al igual que en la actualidad han sido y siguen siendo rechazados por la sociedad. Por lo tanto, es imperativo que la preparación inicial y en su desarrollo como docente se les dé la capacitación para que puedan entender los temas de IPAE, enfocándose en la población de estudiantes más vulnerables en las escuelas americanas.

A causa del impacto de los factores políticos e ideológicos en la educación en los Estados Unidos y la necesidad de resaltarlos estratégicamente en el dialogo de los docentes bilingües se ha centrado la transformación de retos para identificar, nombrar, y enfrentar los aspectos sociopolíticos e ideológicos en la capacitación de los docentes de la educación bilingüe, que deliberadamente se relaciona a los cuatro 
temas de IPAE para crear óptimas condiciones de aprendizaje para todos los estudiantes en programas con instrucción en ambas lenguas (DLE).

\section{La claridad ideológica}

Es esencial que los docentes adquieran un entendimiento firme sobre las ideologías dominantes para que puedan crear discursos contra alocuciones hegemónicas que resisten y transforman prácticas opresoras en las aulas. El proceso de un dialogo crítico, un auto-cuestionamiento continuo y una reflexión donde proviene la evolución es lo que despierta una concientización crítica que conlleva a una claridad ideológica (Darder, 2018; Darder et al., 2017; Freire, 1985; Valenzuela, 2016). Freire (1985) nos indica que una ideología bien articulada de un docente es el faro para empoderarlo/a a navegar por, con, y alrededor de la política que restringe la diversidad lingüística y otras agendas socio-políticas.

Es de suma importancia que los docentes se den cuenta de que su ideología es la que «anuncia o denuncia» su enseñanza por la equidad y justicia social. La claridad ideológica tiene como requisito que las explicaciones individuales de los docentes sean continuamente comparadas y contrastadas con aquellas propagadas por una sociedad dominante (Bartolomé, 2002). Es de salir al camino con la yuxtaposición de ideologías obligue a los docentes a reflexionar de cuando y como su sistema de creencias reflejan de una manera crítica a aquellas de la sociedad dominante y apoyan a las condiciones injustas y desiguales (168). Adicionalmente, Freire (1993) nos recuerda que tanto el aprendizaje como la enseñanza en las escuelas constituye un acto político atado a las fuerzas ideológicas que operan por parte de la clase dominante. Los docentes de DLE deben de resguardarse para no perpetuar las prácticas hegemónicas que han existido en el aula de inglés (monolingüe) y que han llegado a penetrar en el aula de DLE. Los docentes podrán conseguir un desempeño extraordinario dentro de su didáctica para obtener una ideología clara al hacerse las siguientes preguntas críticas:

- ¿Qué creencias, valores y teorías epistemológicas influyen en mis pensamientos?

- ¿Cuáles son las circunstancias políticas, sociales, culturales, lingüísticas, de género y emociones en las cuales yo he aprendido?

- ¿Tengo la valentía para hablar sin reservas en beneficio de mis estudiantes?

- ¿Qué tipo de docente quiero ser?

- ¿Qué quiero que mis estudiantes sepan y hagan bien?

- ¿Qué tipo de cambios debo de implementar en mi docencia para fomentar la identidad bicultural/ bilingüe de mis estudiantes y su desarrollo en su alfabetización literaria?

\section{Perspectiva pedagógica}

Los docentes con una pedagogía clara ejercen una reciprocidad constante de su ideología y didáctica dentro del salón de clases. Una claridad ideológica, una reflexión crítica, y un dialogo transformativo orientan la práctica del docente y la interacción intencional entre la enseñanza y el aprendizaje. La claridad pedagógica de un docente que se deriva desde una perspectiva de conocimiento mutuo se enfoca en la creencia de que los estudiantes y los docentes conceptúen juntos la sabiduría y las experiencias que cada estudiante aporta a la clase (Yosso, 2005). Por lo tanto, los docentes utilizan estrategias para desarrollar el bilingüismo y colaboran con los estudiantes para crear un ambiente democrático dentro del aula. Para que los 
docentes de DLE puedan presenciar una transformación critica en su didáctica, ellos/as deben trabajar diligentemente para desarrollar una claridad ideológica y pedagógica al examinar estas preguntas críticas:

- ¿Qué tipo de valores esenciales e investigaciones rigen mi práctica docente?

- ¿Qué les transmito a mis estudiantes?

- ¿Cómo demuestro mi respeto hacia la riqueza cultural y lingüística de mis estudiantes?

- ¿He creado un ambiente de dialogo dentro del salón o solo les transmito conocimientos?

- ¿Uso los conocimientos de mis estudiantes para que aprendan?

- ¿Respeto el lenguaje coloquial de mis estudiantes mientras les enseño el lenguaje académico?

- ¿Construyo conocimiento con mis estudiantes en un proceso de dialogo?

\section{Acceso para todos}

Con todo lo que sabemos sobre el éxito académico y la motivación de los estudiantes de los programas de DLE (Lindholm-Leary \& Borsato, 2002; Soltero, 2016), claramente existe una escasez de estudios relacionados al acceso a la calidad de currículo, enseñanza y materiales que los estudiantes pueden adquirir. Mientras las normas rigurosas y el vocabulario complejo incrementen las demandas lingüísticas, los docentes de DLE necesitarán recursos adicionales y capacitación para desarrollar las estrategias de enseñanza que garantizan el acceso académico para todos los estudiantes (Hernández, 2011).

Aunque DLE ofrece a los estudiantes latinos las oportunidades para avanzar en sus estudios, puede que los programas produzcan resultados perjudiciales en relación al éxito o al fracaso de los estudiantes de origen mexicano (Valdés, 1997). Algunas posibles desventajas son la calidad de la enseñanza del idioma español, los efectos de la inmersión bilingüe entre las relaciones de los estudiantes, y como DLE afecta el estatus social entre idiomas que afectan a los estudiantes y a nuestra sociedad. Valdés (1997) cuestionó el hecho de que los programas de DLE tienen el potencial de reducir la ventaja natural de los latinos como estudiantes bilingües. Esta es una percepción crítica y valiosa a la cual Valdés nos llamó la atención hace más de dos décadas, y hoy en día se ve claramente. En los programas bilingües compuestos por la clase obrera de habla hispana y los angloparlantes de la clase media, los angloparlantes aprenden la ventaja del español académico y típicamente no se sienten denigrados en el proceso de su aprendizaje. Además, traen consigo las normas culturales de la clase media que pueden transferirse fácilmente para su bien porvenir. Ante esto, el marco de IPAE convoca a los docentes de DLE a reestructurar la noción sociopolítica de acelerar a la clase dominante poderosa y dejar sin poder al grupo subordinado. Como lo vemos, si realmente tomamos con seriedad el hecho de exigir igualdad de condiciones dentro del ámbito educativo, es imperativo que los docentes bilingües, quienes enseñan a estudiantes de los niveles económicos más humildes en nuestra sociedad que intencionalmente se resistan e interrumpan persistentemente las pedagogías hegemónicas (Cervantes-Soon et al., 2017; Quezada \& Alfaro, 2012).

La problemática de utilizar más inglés en los programas bilingües para obtener mejores resultados en las pruebas normalizadas al reducir el tiempo con la lengua materna es una preocupación para muchos docentes. Lindholm-Leary and Genesee (2010) explicaron que esta táctica no tiene fundamento científico que apoye su funcionamiento. Al contrario, al añadir más inglés en ciertas actividades no agiliza la adquisición del inglés ni mejora el puntaje o promedio de los estudiantes (Gándara \& Hopkins, 2010). Se 
requiere un profundo análisis para poder entender los conceptos fundamentales del tercer tema de IPAE, el acceso. Las siguientes preguntas son para reflecionar como es que el idioma y el poder encajan dentro del aula:

- ¿A quién le presto mi atención?

- ¿Estoy estratégicamente creando un acceso universal para todos mis estudiantes?

- ¿A quién elijo primero para responder a las preguntas de apredizaje?

- ¿Quiénes son mis estudiantes de mayor rendimiento?

- ¿Quiénes necesitan ayuda adicional?

- ¿Estoy bajando los estándares de enseñanza y aprendizaje?

- ¿Estoy aplicando rigor y expectativas altas para todos mis estudiantes?

\section{Entornos equitativos}

El tema de IPAE sobre la equidad se define como la base de la justicia social dentro del aula de DLE, incluyendo como definimos y posicionamos las metas sociolingüísticas y socioculturales para todos los estudiantes. La equidad le da a los docentes de DLE un lente para filtrar como ellos pueden aplicar su claridad ideológica para crear entornos democráticos y seguros, examinar la participación de los miembros del grupo y balancear el estatus del idioma con la didáctica. No es nada fácil para un docente bilingüe mantener la equidad social dentro del salón ya que el idioma inglés es el idioma que representa el poder en los Estados Unidos. Aunque los programas de DLE afirman una meta intercultural, los estudios sobre el estatus de la cultura y la lingüística entre español e inglés (Alfaro \& Bartolomé, 2017; De Jong, 2006; Fitts, 2006; Hernández, 2011; Palmer, 2008) afirman las cuestiones en relación a la justicia social y la equidad. Los resultados indican que los angloparlantes le faltan el respeto a los hispanohablantes en el ambiente académico al interrumpir a sus compañeros en discusiones e impedir su contribución oral dentro del salón de clases (Hernández, 2011; Palmer, 2008; Palmer \& Martínez, 2013).

Potowski (2004) estudió factores que influyen la importancia del idioma inglés y la eliminación de algunas conexiones valiosas al idioma de los hispanohablantes en las escuelas de DLE. Estas atribuciones incluyen el énfasis del idioma inglés en los exámenes normalizados, clases optativas, asambleas, deportes, ferias y otras prácticas escolares que se llevan a cabo en inglés que claramente mandan mensajes sutiles de superioridad lingüística a los estudiantes, mientras ellos empiezan a asociar el estatus de poder con el idioma dominante de la escuela. Los hispanohablantes pueden tener la tendencia a consentirse con el idioma dominante de sus compañeros para afirmar su estatus y competencia lingüística en inglés, hasta sucede con los recién llegados que saben muy poco inglés. Potowski notificó que durante la enseñanza de español, el 68\% del tiempo dedicado a las conversaciones de los estudiantes resultó en inglés mientras que las conversaciones en español solo se dieron en un 32\%. Una vez más, tenemos que enfrentarnos a las preguntas difíciles en cuanto a la equidad:

- Honestamente, ¿De qué manera soy un modelo a seguir con el idioma?

- ¿Quién es dominante en las discusiones en clase?

- ¿De qué manera estoy navegando el estatus de cultura e idioma dentro del aula? 
- ¿De qué manera perciben los estudiantes el idioma inglés?

- ¿Algunos de mis estudiantes se están conformando con un rol subordinado? ¿Por qué?

- ¿De qué manera están participando los estudiantes en el idioma de enseñanza?

- ¿De qué manera las evaluaciones le están dando mayor enfoque al uso de los idiomas dentro del salón?

- ¿De qué manera influyen las actividades dentro del salón y la escuela al poder que se le da al idioma dominante o grupo?

\section{6. Últimas palabras}

La preferencia al uso del inglés durante la clase de español afecta la riqueza lingüística de los hispanohablantes y marchita la vitalidad del idioma español dentro del salón de clases (Hernández, 2011, 2015). Los docentes de DLE tienen que ser conscientes de la preferencia del idioma durante las actividades y encontrar maneras para mantener la equidad lingǘstica. Dado que el idioma y la cognición son elementos centrales para desarrollar el bilingüismo y la alfabetización literaria en el entorno de DLE, los docentes deben crear oportunidades innovadoras y con el propósito de usar la lengua de destino (ej. español) y deben encontrar maneras para mantener las conversaciones sin cambiar de idioma de una manera que favorezca el idioma dominante y disminuya al español. Por consiguiente, simplemente no es suficiente organizar a los estudiantes para que hagan trabajos en español (ej. trabajo en equipos/grupos, círculos literarios) eso no garantiza la comunicación académica en español. Los docentes pueden manipular estos paradigmas por medio de discursos alternativos/un uso intencional del idioma (Palmer, 2008), supervisar el uso del idioma (De Jong, 2006), buscar aliados de español para las actividades en equipo que mantengan las conversaciones en español (Fitts, 2006), usar métodos para demostrar estructuras gramaticales (Lyster, 2009), enfatizar como enriquecer el lenguaje escrito (Hernández, 2011; 2015), y darle valor a la identidad cultural y propósito para el uso de la lengua que motivan el automatismo y elocuencia en español (Norton, como fue citado en Potowski, 2004).

El crecimiento sumamente rápido de los programas de DLE en los Estados Unidos ha hecho más evidente la apremiante necesidad de auto examinar las prácticas que son críticamente conscientes y fundamentales a la equidad y justicia social. Al estudiar los cuatro temas, Ideología, Pedagogía, Acceso y Equidad (IPAE), nos permite cuestionar lo que nos ayuda a reflexionar y a reaccionar la parte fundamental de IPAE. Por esta razón, hemos desarrollado una guía de preguntas para ayudar a los docentes a reflecionar y examinar sus pensamientos y su práctica en el aula.

\section{Conclusión}

Quedamos firmes en que la equidad educativa sucede cuando las prácticas educativas, las políticas, los planes de estudio, los recursos y las culturas escolares son representativos de todos los estudiantes, sus familias y comunidad de modo que cada alumno tenga acceso, pueda participar y progresar en experiencias de aprendizaje de alta calidad, en ambos idiomas, independientemente de su identidad cultural, diversidad lingüística, nivel socio-económico, género, capacidad, religión, y origen nacional. La conciencia crítica de los docentes es necesaria para abogar por sus estudiantes y cuestionar las políticas restrictivas y opresivas de educación en los programas de DLE (Cervantes-Soon, et al., 2017; Freire, 2016). Dado esto, el desarrollo de la conciencia crítica del docente necesita una claridad ideológica que incluya un autoexamen que conlle- 
ve la capacidad de reconocer la diferencia en las relaciones de poder en el aula entre estudiantes, docentes, familias, comunidades y entre fronteras. En la misma línea, los docentes deben analizar críticamente quién y qué informa la ideología y la pedagogía que pueden potencialmente «anunciar o denunciar» la igualdad en el aula. Es esencial que docentes de DLE desarrollen una voz académica que, con valentía e inteligencia, aborde cuestiones de justicia social desde una postura cultural, lingüística y anclada en la comunidad. Docentes críticos deben trabajar desde una perspectiva basada en los activos que honre, respete y valore a los estudiantes, sus historias, familias y comunidades. Sin fomentar intencionalmente los fondos de identidad de los estudiantes honrando la riqueza de la cultura de los estudiantes, fondos de conocimiento, variedades de idiomas y habilidades lingüísticas, los estudiantes no lograrán completa y aditivamente discursos académicos tanto en inglés como en el idioma asociado en espacios equitativos de dos idiomas.

Dada la ideología histórica y profundamente enraizada al monolingüismo en inglés y a las practicas asimilistas en cuanto a los estudiantes de una lingüística minorizada, se insiste que la capacitación del docente de DLE debe explícitamente abordar el rol de la claridad ideológica, tener una crítica perspectiva pedagógica, darle acceso a todos sus estudiantes, y centralizarse en la equidad al crear el currículo, ya que el hecho de que las relaciones asimétricas del poder están entre la preferencia al idioma de la clase media anglosajona o desarrollar ambas lenguas académicamente (García, 2009; Lewis et al., 2013; Sayer, 2013). En DLE, es particularmente importante capacitar al docente para que se dé cuenta de sus propias preferencias hacia las normas de la clase media, incluyendo sus propios prejuicios en contra del idioma que a veces no se considera académico (ej. español).

De acuerdo con Soltero (2016) el primer y más importante elemento fundamental para una educación eficaz en dos idiomas (DLE) se basa en docentes bien informados y bien preparados. Se puede decir que la mayoría de los estudios para capacitar a los docentes de DLE se enfocan en impartir los conocimientos técnicos, tales como la variedad de metodologías que los ayudaran a desarrollar la experiencia en una serie de ámbitos para impartir la enseñanza bilingüe (Wright et al., 2015). Creemos firmemente que es tan importante el conocimiento del docente en estas habilidades metodológicas tanto como el conocimiento del docente en la ideología crítica que desarrolla su pedagogía y que crea acceso para estudiantes de diversas culturas para asegurar equidad dentro del aula de DLE. Nos suscribimos al marco teórico que cuestiona la noción del desarrollo de la lectoescritura como una construcción monolítica. Al contrario, lo consideramos como un balance de los poderes asimétricos integrado en la complejidad educativa de las relaciones socioculturales, tensiones, posibilidades y oportunidades.

\section{Referencias bibliográficas}

Alfaro, Cristina (2017): “Growing Ideologically Clear and Linguistically Efficacious Dual Language Teachers". The Multilingual Educator, 36-40. Retrieved from the California Association for Bilingual Education website: http://www.gocabe.org/index.php/communications/multilingual-educator/.

Alfaro, Cristina \& Bartolomé, Lilia (2018): "Preparing Ideologically Clear Bilingual Teachers to Recognize Linguistic Geniuses" in B. Berriz, A. Wagner, \& V. Poey (eds): Art as a Way of Talking for Emergent Bilingual Youth: A Foundation for Literacy in K-12 Schools. New York, NY: Routledge/Taylor and Francis.

Alfaro, Cristina \& Bartolomé, Lilia (2017): "Preparing Ideologically Clear Bilingual Teachers: Honoring Working-class Non-standard Language use in the Bilingual Education Classroom". Issues in Teacher Education, 26 (2), 11-34. 
Alfaro, Cristina; Durán, R.; Hunt, A.; \& Aragón, M.J. (2015): “Steps Toward Unifying Dual Language Programs, Common Core State Standards, and Critical Pedagogy". Association of Mexican American Educators Open Issue, 8 (2), 17-30.

Alfaro, Cristina (2008). “Teacher Education Examining Beliefs, Orientations, Ideologies \& Practices” in L. Bartolome (ed.): Ideologies in Education: Unmasking the Trap of Teacher Neutrality. New York, NY: Peter Lang Publishing Group.

Baker, Colin (2011). Foundations of bilingual education and bilingualism ( $5^{\text {th }}$ edition). Clevedon, England: Multilingual Matters.

Bartolomé, Lilia (2010): "Daring to Infuse Ideology into Language-teacher Education” in S. May, \& C. Sleeter (eds.): Critical Multiculturalism: From Theory to Practice. New York, NY: Routledge.

Bartolomé, Lilia (2008). "Beyond the Fog of Ideology" in L. Bartolomé (ed.): Ideologies in Education: Unmasking the Trap of Teacher Neutrality. New York, NY: Peter Lang Publishing Group.

Bartolomé, Lilia (2002). "Creating an Equal Playing Field: Teachers as Advocates, Cultural Border Crossers, and Cultural Brokers" in Z. Beykont (ed.): The Power of Culture: Teaching Across Language Differences. Cambridge, MA: Harvard Education.

Callahan, Rebecca \& Gándara. Patricia (Eds.). (2014). The Bilingual Advantage Language, Literacy and the US Labor Market. Buffalo, NY: Multilingual Matters.

Cazden, Courtney (2001). Classroom Discourse: The Language of Teaching and Learning (2 ${ }^{\text {nd }}$ Edition). Portsmouth, NH: Heineman.

Cervantes-Soon, C. G.; Dorner, L.; Palmer, D.; Heiman, D.; Schwerdtfeger, R., \& Choi, J. (2017): “Combating Inequalities in Two-Way Language Immersion Programs: Toward Critical Consciousness in Bilingual Education Spaces". Review of Research in Education, 41, 403-427.

Darder, Antonia (2018). The Student Guide to Freire's Pedagogy of the Oppressed. London, UK: Bloomsbury Academic.

Darder, Antonia (2015). Freire and Education. New York, NY: Routledge.

Darder, Antonia; Torres, Rodolfo D., \& Baltodano, Marta (Eds.). (2017). The Critical Pedagogy Reader (3 ${ }^{\text {rd }}$ ed.). New York, NY: Routledge.

Darder, Antonia, Baltodano, Marta, \& Torres, Rodolfo D. (2003): "Critical Pedagogy: An Introduction" in Antonia Darder, Marta Baltodano \& Rodolfo D. Torres (eds.): The Critical Pedagogy Reader. New York, NY: Routledge Falmer.

Freire, J.A. (2016): "Nepantleras/os and their Teachers in Dual Language Education: Developing Sociopolitical Consciousness to Contest Language Education Policies". Association of Mexican American Educators Journal, 10 (1).

Freire, Paulo (1993). Pedagogy of the City. New York, NY: Continuum.

Freire, Paulo (1985). The Politics of Education: Culture, Power, and Liberation. New York, NY: Bergin \& Garvey.

De Jong, E. (2006): “Integrated Bilingual Education: An Alternative Approach”. Bilingual Research Journal, $30(1), 23-44$. 
Fitts, S. (2006): "Reconstructing the Status Quo: Linguistic Interaction in a Dual-Language School". Bilingual Research Journal, 29 (2), 337-365.

Gándara, Patricia \& Maxwell, Jolly, Julie (2000). Preparing Teachers for Diversity: A Dilemma of Quality and Quantity. Santa Cruz, CA: Center for the Future of Teaching and Learning.

Gándara, Patricia \& Hopkins, Megan(2010). Forbidden Languages: English Learners and Restrictive Language Policies. New York, NY: Teachers College Press.

García, Ofelia (2009). Bilingual Education in the 21t Century: A Global Perspective. Oxford, UK: Wiley-Blackwell.

García, Ofelia (2014): "U.S. Spanish and Education: Global and Local Intersections” in K.M. Borman, T.G. Wiley, D.R. García, \& A.B. Danzig (eds.): Review of Research in Education: Language Policy, Politics, and Diversity in Education, 38 (1), 58-80.

Hernández, Ana M. (2015): "Language Status in Dual Immersion: The Dynamics Between English and Spanish in Peer Interaction”. Journal of Immersion and Content-Based Language Education, 3 (1), 102-126. DOI: https://doi.org/10.1075/jicb.3.1.05her (C) John Benjamins Publishing Company.

Hernández, Ana M. (2011). Successes and Challenges of Instructional Strategies in Two-Way Bilingual Immersion. Unpublished Doctoral Dissertation. San Diego, CA: University of California.

Howard, Elizabeth R.; Sugarman, Julie, \& Christian, Donna (2003). Trends in Two-Way Immersion Education: A review of the literature (Report No. 63). Baltimore, MD: Center for Research on the Education of Students Placed At Risk.

Howard, Elizabeth R.; Lindholm-Leary, Kathryn J.; Rogers, David; Olague, N., Medina, J., Kennedy, D., Christian, Donna (2018). Guiding Principles for Dual Language Education $\left(3^{\text {rd }}\right.$ ed.). Washington, DC: Center for Applied Linguistics.

Lewis, G., Jones, B., \& Baker, C. (2013): “100 Bilingual Lessons: Distributing Two Languages in Classrooms" in C. Abello-Contesse \& R. Chacon Beltran (eds.): Bilingualism in a School Setting. Bristol, UK: Multilingual Matters.

Lindholm-Leary, Kathryn J. (2001). Dual Language Education. Clevedon, England: Multilingual Matters.

Lindholm-Leary, Kathryn J., \& Genesee, F. (2010). "Alternative Educational Programs for English Learners. In California Department of Education” (eds.): Improving Education for English Learners: ResearchBased Approaches. Sacramento, CA: CDE Press.

Lindholm-Leary, Kathryn J., \& Howard, E. R. (2008). "Language Development and Academic Achievement in Two-Way Immersion Programs" in T. Williams Fortune and Diane J. Tedick (eds.): Pathways to Multilingualism: Evolving Perspectives on Immersion Education. Clevedon, England: Multilingual Matters.

Lindholm-Leary, Kathryn J., \& Borsato, G. (2002). Impact on Two-Way Immersion on Students' Attitudes Toward School and College (Report No.10). Washington, DC: Center for Research on Education, Diversity and Excellence. (ERIC Document Reproduction Service No. ED-99-CO-0008).

Lyster, Roy (2007). Learning and Teaching Languages Through Content: A Counterbalanced Approach. Philadelphia: John Benjamins. 
Palmer, D. K. (2008). "Diversity Up Close: Building Alternative Discourses in the Two-Way Immersion Classroom" in T. Williams Fortune and Diane J. Tedick (eds.): Pathways to Multilingualism: Evolving Perspectives on Immersion Education. Clevedon, England: Multilingual Matters.

Palmer, D. \& Martínez, R. A. (2013): "Teacher Agency in Bilingual Spaces: A Fresh Look at Preparing Teachers to Educate Latina/o Bilingual Children”. Review of Research in Education, 37, 269-298.

Potowski, Kim (2004): "Student Spanish Use and Investment in a Dual Immersion Classroom: Implications for Second Language Acquisition and Heritage Language Maintenance". Modern Language Journal, 88, 75-101.

Quezada, R. \& Alfaro, Cristina (2012). "Cutting to the Common Core: Moving Pedagogic Mountains". Language Magazine, 11 (12), 19-22.

Soltero, S. W. (2016). Dual Language Education: Program Design and Implementation. Portsmouth, NH: Heinemann.

Sugarman, Julie, \& Howard, Elizabeth R. (2001). Development and Maintenance of Two-Way Immersion Programs: Advice from Practitioners. Practitioner Brief \#2. Santa Cruz: Center for Research on Education, Diversity \& Excellence, University of California. (Cooperative Agreement No. R306A60001-96).

Valdés, Guadalupe (1997): "Dual Language Immersion Programs: A Cautionary Note Concerning the Education of Language-Minority Students”. Harvard Educational Review, 67 (3), 391-429.

Valenzuela, Angela (Ed.). (2016). Growing Critically Conscious Teachers: A Social Justice Curriculum for Educators of Latino/a Youth. New York, NY: Teachers College Press.

Wright, Wayne E.; Boun, Sovicheth \& Garcia, Ofelia (2015). The Handbook of Bilingual and Multilingual Education. NJ: Wiley.

Yosso, Tara J. (2005). "Whose Culture has Capital? A Critical Race Theory Discussion of Community Cultural Wealth". Race, Ethnicity, and Education, 8(1), 69-91.

\section{Notas biográficas}

Cristina Alfaro is Professor in the College of Education at San Diego State University in the Dual Language and English Learner Education Department. For more than 30 years, her practice and scholarship have focused on political and ideological questions and ethical concerns linked to class inequalities, language rights, and social justice. She has over two decades of experience in directing both local and international bilingual teacher education programs in California, Mexico, and Europe.

Ana Hernández is Associate Professor and Program Coordinator of Multilingual and Multicultural Education in the School of Education at California State University San Marcos. Her work in bilingual teacher preparation stems from 32 years of teaching in California public schools as a bilingual/dual language teacher in grades K-8th. Dr. Hernández's research examines instructional practices and crosscultural equity in dual language education. 